\title{
Short- and Long-Term Components of Working Memory in the Rat
}

\author{
Sheri J. Y. Mizumori, Veena Channon, \\ Mark R. Rosenzweig, and Edward L. Bennett \\ University of California, Berkeley
}

\begin{abstract}
Previous experiments suggested that working memory of rats trained on a radial maze can be discussed in terms of its short- and long-term temporal components. For example, in Mizumori, Channon, Rosenzweig, and Rennett's (1985) study, long-term working memory was found to be susceptible to distuption by the protein synthesis inhibitor anisomycin (ANI). In Experiment 1 of this report, we examined the neuropharmacological nature of short-term working memory of rats trained to retrieve food from all arms of a 12-arm radial maze. Delay intervals of varying length were placed between Choices 6 and 7 . Lanthanum $\left(\mathrm{LaCl}_{3}\right)$ and glutamate (GLU) injected bilaterally into the hippocampus effectively impaired retention over short delay intervals, which suggests a possible role for calcium and/or potassium and for glutamate in working memory. However, another equally likely explanation for the amnesic effects of $\mathrm{LaCl}_{3}$ and $\mathrm{GLU}$ is that these drugs impaired reference memory. To test more directly the hypothesis that $\mathrm{LaCl}_{3}$. GLU. or ANI might differentially affect working and reference memory, we tested the efferts of these drugs on performance of rats trained to retrieve food from only 8 arms of the 12-arm maze in Experiment 2. The remaining 4 arms were never baited, in order to test reference memory function. We predicted that rats would make errors only in baited arms (i.e., errors of working memory). Instead, results of Experiment 2 showed that $\mathrm{LaCl}_{3}$. GLU. or $\mathrm{ANI}$ injection produced errors in unbaited arms even before a 120-min delay. If rats were injected with $\mathrm{LaCl}_{3}$ or GLU, baited-arm errors were observed only after the delay period. No impairment of performance on baited arms were observed after injection of ANI. These data are discussed in terms of the possible task-dependent nature of the relative contribution of different chemical systems to different memory processes.
\end{abstract}

Several lines of research suggest that the formation of longterm memories involves the synthesis of new proteins (see Davis \& Squire. 1984, for review). Administration of protein synthesis inhibitors near the time of behavioral training results in amnesia hours to days later in rats (e.g., Daniels, 1972), mice (e.g., Cohen \& Barondes, 1968), and chicks (Gibbs \& $\mathrm{Ng}$. 1976), as well as in invertebrates such as Aplysia (Montarolo, Castellucci, Goelet, Kandel, \& Schacher, 1985) and cockroaches (Barroco. Lovell, \& Eisenstein, 1981). Evidence for the involvement of particular chemical systems in shortterm memories is less clear. Perhaps one of the most extensive examinations of the neurochemical basis of retention over relatively short periods of time was carried out by Gibbs and colleagues (see Gibbs \& Ng, 1977). The experimental paradigm used for most of Gibb's studies involved intracranial injections of various classes of pharmacological agents close to the time of one-trial taste avoidance training in chicks. Retention was tested at various times after training, from

The research reported here was supported by Grant $1 \mathrm{ROI}$ MH36042 from the National Institute of Mental Health to M. R. Rosenzweig and E. L. Bennett. The authors greatly appreciate the assistance of Helene Blaustein, Shawnya Kaleta, Mike Paff, and Matthew Pease with behavioral testing and data analysis.

Correspondence concerning this article should be addressed to Sheri J. Y. Mizumori, who is now at the Department of Psychology, Behavioral Neuroscience Area, Campus Box 345, University of Colorado. Boulder, Colorado 80309. minutes to hours later. On the basis of their results. Gibbs and $\mathrm{Ng}$ hypothesized that memories are formed in three temporally sequential and neurochemically distinct phases. The shortest lasting phase (referred to by Gibbs and $\mathrm{Ng}$ as short-term memory, or STM) persists for about $5-15 \mathrm{~min}$ after training on their task and is susceptible to disruption or enhancement by drugs that affect $\mathrm{K}+$ or $\mathrm{Ca}+2$ conductance across neuronal membranes (e.g., $\mathrm{KCl}$, glutamate, or $\mathrm{CaCl}_{2}$ : Gibbs. Gibbs \& Ng. 1978, 1979: Gibbs \& Ng. 1979). An intermediate-term memory (ITM) was postulated to last approximately $15-30$ min after training and is supported neurochemically by the $\mathrm{Na}+\mathrm{K}+$ ATPase. Drugs that influence this ATPase, such as ouabain or diphenylhydantoin, could modulate the duration of ITM (Gibbs \& Ng. 1984a; Watts \& Mark, 1971). Finally, long-term memories (I.TM)-memories that last longer than $30 \mathrm{~min}$-require protein synthesis and are susceptible to disruption by different protein synthesis inhibitors (Gibbs \& Ng, 1984b: Watts \& Mark, 1971).

Although the data presented in the many reports by Gibbs and colleagues are internally consistent, the implications of their data must be considered carefully. The fact that the length of good retention of prior avoidance training varied according to the drug injected does not necessarily imply that the drugs were affecting psychologically different types of memories, as was implicated by their use of the terms STM. ITM, and LTM. The behavior task used by Gibbs and $\mathrm{Ng}$ did not allow the determination of which type of cognitive or memory process was affected by the different classes of drugs. 
To examine this question, we began a series of investigations to determine the effects of the same drugs used by Gibbs et al. $(1978,1979)$ on performance of rats in a task that allows clearer behavioral definition of the type of memory used, the radial maze.

Rats can be trained to retrieve food pellets placed at the ends of arms of a 12-arm radial maze by entering each arm only once in a daily training session. Perfect performance by the rat, therefore, involves making 12 different choices in a given training session. Delays of several hours can then be interposed between Choices 6 and 7. Olton $(1978,1983)$ claimed that this training procedure allows one to examine the effects of drugs on two psychologically distinct memory systems: working and reference memories. Working memory items are thought to be specific in nature and are typically applied to only one occasion. Such items are nol stored for long periods of time. Reference memory items, on the other hand, include more general information than working memory. Associations regarding particular rules and procedures necessary for appropriate interactions with one's environment involve reference memory. Such associations can be used on many occasions and can be stored for relatively long periods of time. Because the first six choices of a training session vary from day to day, retention of these choices over an interposed delay interval indicates good working memory abilities. A rat can demonstrate good reference memory if it remembers the rules of the maze. For example, the rat should readily run down arms to retrieve the food pellets. then return to the center platform to make the next choice. The training procedure can be altered in such a way that one can test reference memory more directly by converting a subset of the available arms to ones that never contain food. Rats will soon learn to never enter these arms because the unbaited set of arms is the same from day to day. The information regarding the significance of these unbaited arms is thought to involve reference memory. Therefore, when a well-trained rat enters these unbaited arms, it may be because of deficiencies in its reference memory system.

Results of a recent study that involved behavior testing of rats on the radial maze (Mizumori, Rosenzweig, \& Bennett, 1985) suggested that working memory items that are to be held in store over a relatively long delay period (i.c., greater than $15 \mathrm{~min}$ ) may involve the synthesis of ncw proteins. In that study, rats were trained to retrieve food placed at the end of each of 12 arms of the maze with 240-min delays between Choices $1-6$ and Choices 7-12. After the rats achieved asymptotic performance levels, a protein synthesis inhibitor. anisomycin (ANI), was injected bilaterally through indwelling cannulae into the hippocampus, an area of the brain thought to be critical for accurate performance in this task (c.g., Handelmann \& Olton, 1981). After injection of ANI, significant impairment of retention over delays of $15 \mathrm{~min}$ or longer was observed. Choice accuracy was not impaired if $0-$ or 2-min delays were used. No attempt was made to examine the neurochemical nature of retention of working memory items over very short periods of time (i.e., less than $15 \mathrm{~min}$ ).

On the basis of Gibbs and $\mathrm{Ng}$ 's (1977) hypothesis regarding the role of $\mathrm{K}+$ and/or $\mathrm{Ca}+2$ in memories of short duration, one might expect that $\mathrm{K}+$ or $\mathrm{Ca}+2$ plays a significant role in short-term working memories. Indeed, there are indications that normal calcium function is important for working memory processes. Davis, Idowu, and Gibson (1983) reported that injection of 3,4-diaminopyridine, a facilitator of calcium uptake into cells, improved radial maze performance of aged rats. The role of calcium in working memory has also been studied indirectly by Staubli, Baudry. and Lynch (1984). They implanted into rats osmotic pumps that permitted continuous subcutaneous infusion of leupeptin, an inhibitor of a calciumdependent proteinase. Staubli et al. found that leupeptin impaired radial maze performance when delays of $1 \mathrm{~min}$ to 1 $\mathrm{hr}$ were interposed between choices. In Experiment 1 of this report. we examined whether the chemical systems that Gibbs and $\mathrm{Ng}(1977)$ held to be responsible for short-lasting memories- $\mathrm{K}+$ or $\mathrm{Ca}+2$ conductance-might play an important role in retention of working memory items over relatively short delay periods. For this experiment, glutamate (GLU) or lanthanum ( $\mathrm{LaCl}_{3}$, which blocks $\mathrm{Ca}+2$ uptake) was injected bilaterally into the hippocampus of rats. In Experiment 2 we examined the question of whether the $\mathrm{LaCl}_{3}$ - or GLU-induced deficits observed in Experiment 1 were attributable to the drugs' effects on a short-term component of working memory or to an impairment in reference memory. For both of these experiments, an ANI-injected treatment condition was included for comparison.

\section{Experiment 1}

\section{Method}

Subjects. Seventy-day-old Long-Evans rats were obtained from Charles River Breeding Laboratory (Portage. Michigan for Experiment 1: Kingston. New York for Experiment 2). After 1 week of adaptation to the laboratory environment, the rats were placed on food restriction to maintain their body weights at $80 \%-85 \%$ of their ad lib body weights. Lights were on in the colony room between 1900 and $0700 \mathrm{hr}$. Behavioral testing occurred during the dark phase of the light/dark cycle, between 0800 and $1400 \mathrm{hr}$.

Apparatus. The rats were tested on the same elevated radial maze described previously by Mizumori. Rosenzweig, and Bennett (1985). Briefly, 12 arms $(80 \times 10.2 \mathrm{~cm}$ and $61 \mathrm{~cm}$ above the floor) extended from a center platform $(40.6 \mathrm{~cm}$ in diameter). Access to individua! arms was controlled by opaque Plexiglas doors that were operated by the experimenter via a system of fishline. Several extramaze room cues, such as chairs, air vents, posters. and the experimenter, were available.

Drug preparation. Lanthanum chloride $\left(\mathrm{LaCl}_{3}\right)$ and glutamate (GLU) were obtained from Sigma Chemical Company. $\mathrm{LaCl}_{3}$ was dissolved in saline: the $\mathrm{pH}$ was adjusted to 7.4 by the addition of $\mathrm{NaOH}$. GLU was dissolved in saline. with no $\mathrm{pH}$ adjustment. The concentrations of $\mathrm{LaCl}_{3}$ and GLU were 14.8 and $187 \mu \mathrm{g} / \mu \mathrm{l}$. respectively. Preliminary experiments indicated these doses to be the smallest that one could inject and still obtain reliable amnesia. Anisomycin (2-p-methoxyphenyl-3-acetoxy-4-hydropyrollidine) was obtained from Warner-Lambert, General Diagnostics Division: we dissolved it in saline by adding equal molar amounts of $3 \mathrm{~N} \mathrm{HCl}$. The $\mathrm{pH}$ was adjusted to 7.4 by the addition of $\mathrm{NaOH}$. We used $80 \mu \mathrm{g} / \mu \mathrm{l} \mathrm{AVI}$ to induce amnesia; this dose was shown to be effective in previous experiments. A volume of $0.5 \mu \mathrm{l}$ was injected per hemisphere.

Surgical procedures. We surgically implanted chronic indwelling cannulae into rats as described previously (Mizumori. Rosenzweig. 
\& Bennett, 1985). Briefly, two stainless steel guide cannulae (23 gauge) were positioned so that the drug could be applied directly to ventral hippocampus (stereotaxic coordinates from bregma: A.P -4.8, L \pm 4.8 , and D-V -6.3 , horizontal skull). After surgery, 0.05$\mathrm{ml}$ Bicillin was injected into cach hind leg to guard against infection. For drug injection, the rats were lightly anesthetized with Mctofanc (methoxyflurane), then loosely placed in a stereotaxic instrument. $\mathrm{LaCl}_{3 *} \mathrm{GLU}, \mathrm{ANI}$, or saline (SAL) were injected through 31 -gauge blunt needles over a period of $88 \mathrm{~s}$ by an automatic syringe pump. (In more recent experiments. administration of these drugs to awaken animals produced similar behavioral results, as will be reported. $\Lambda$ fter behavioral testing, the brains were sectioned for histological verification of cannula placement.

Training procedure. Five rats were trained to perform accurately on the radial maze according to the procedure outlined in an earlier report (Mizumori, Rosenzweig. \& Bennett, 1985). In particular, the rats learned to obtain food pellets (two $45-\mathrm{mg}$ Noyes pellets) from the ends of the arms by visiting each arm only once in a given (daily) training session. Each rat was confined to the center platform for $5 \mathrm{~s}$ between successive choices. This confinement procedure was intended to reduce the contribution of a response bias to arm selection. After 7 days of criterion performance (i.e., making at least 11 correct. or different, choices in the first 12 choices of the training session and choosing all 12 arms within the first 14 choices), each rat was trained to perform correctly with progressively longer delay intervals between Choices 6 and 7 . Delay intervals of 1,2,15,30,60, 120, and 240 min were used. After each rat reached criterion performance for 3 consecutive days with 240 -min delays, presurgical ad-lib access to food was allowed for 7 days. Food was removed the day before surgery, then replaced after cannula implantation for an additional 7 days.

After again achieving criterion performance with 240 -min delays during the postsurgical training period, the rats were injected on different days with either SAL, $\mathrm{LaCl}_{3}$, GLU, or ANI $30 \mathrm{~min}$ before Choice I. Results of an earlier experiment indicated injection of these drugs during the delay period was ineffective in producing amnesia (Mizumori, Channon, Rosenzweig, \& Bennett, 1985). We tested the eflect of drug injection by using 0 - 2- 8. . or $15 \cdot \mathrm{min}$ delays after Choice 6 . When tested under the 0 -min condition, the rat was allowed to retrieve food from all 12 arms without interuption by the experimenter. The order of drug injected and delay interval tested for each rat was determined as follows: Each combination of drug injected and delay interval lested was considered one treatment condition of the experiment. For each rat, we assigned a randomly determined (without replacement) series of treatment conditions by using a random-numbers table. Each rat underwent a different serial order of treatments. In the end, each rat participated once in cach of the possible treatment conditions. The days of injection were separated by 3 consecutive days of criterion performance by the rat. The experimenter was blind to the drug condition of the animal.

\section{Results and Discussion}

Upon histological examination of injection sites, we found that the tip of the injection needle was localized to within 0.3 $\mathrm{mm}$ of the CA3 portion of ventral hippocampus for all five rats. Injection of any drug (or SAL) did not impair the accuracy of Choices 1-6 (predelay choices). In contrast. postdelay performance after injection of either $\mathrm{LaCl}_{3}, \mathrm{GLU}$, or ANI was generally significantly worse than when the rats were injected with SAL (see Figure 1). A repeated-measures analysis of variance (ANOVA) revealed a significant main effect of drug, $F(3,12)=11.00, p<.01$, with no main effect of delay

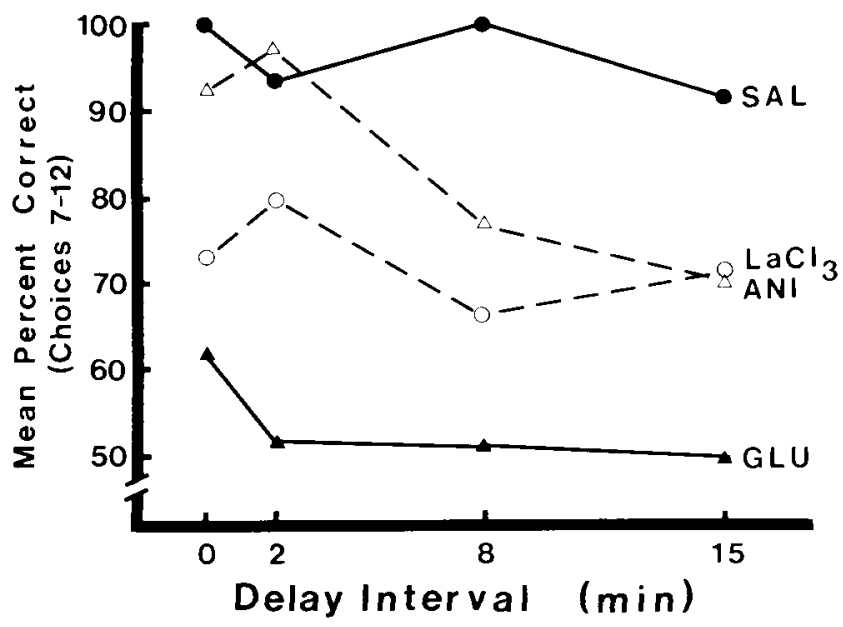

Figure 1. Choice accuracy after 0 - to 15 -min delay intervals between Choices 6 and 7. [Whereas control rats performed well at all delay intervals tested. lanthanum ( $\mathrm{LaCl}_{3}: n=5$ ), glutamate ( $\mathrm{GLU}: n=5$ ). and anisomycin (ANI; $n=5$ ) injection resulted in significant impairment after a 15 -min delay. All drugs impaired performance when 8 min delays were inposed. However, when 0 - or 2-min delays were used, $\Lambda$ NI-treated rats performed as well as controls. No such interaction effect between drug and delay interval was observed if $\mathrm{LaCl}_{3}$ or GI.U was injected. SAL = saline.]

interval. $F(3.12)=2.34 . p>05$. There was no overall interaction effect between drug treatment and delay interval. $F(9,36)=1.16, p>.05$. When individual comparisons were made between the effects of ANI and SAL. not only was a significant drug effect found, $F(1,4)=18.96, p<01$, but, in addition, there was a significant effect of delay interval, $H(3$. $12)=3.69, p<.05$, and a significant interaction effect between drug treatment and delay interval, $F(3,12)=7.56, p<.01$. As reported previously (Mizumori. Rosenzweig. \& Bennett. 1985), ANI-treated rats performed well with delays of up to 2 min; by 15 -min posttraining. amnesia was evident. The results of this experiment confirmed our earlier report and, in addition. revealed that ANI-induced amnesia develops as early as 8 min after training.

A repeated-measures a NOVa also revealed a significant effect of $\mathrm{LaCl}_{3}$ or GLU injection on performance after a delay (see Figure 1), $F(1,4)=10.96, p<.05$ and $F(1,4)=25.00$. $p<.01$. respectively. No effect of delay interval per se was found either for comparisons of $\mathrm{LaCl}_{3}$ or for GLU. $H(3,12)$ $=0.23, p>.05$ and $F(3,12)=1.00 . p>.05$. respectively. However, in contrast to the effects of ANI. neither $\mathrm{LaCl}_{3}$ or GLU injection produced behavioral deficits that were dependent on the length of the delay interval. $F(3,12)=0.53$. $p>.05$ and $F(3,12)=0.52, p>05$. respectively.

Upon examination of the total number of errors required to retrieve the food from all 12 arms (see Figure 2), it was clear that for the most part. significantly more errors were made on days when ANI. GLU, or $\mathrm{LaCl}_{3}$ was injected than on days when SAL was injected, $F(3.12)=24.53 . p<.001$. Comparisons between the effects of SAL and ANI revealed a significant effect of ANI on the number of errors made. $F(1$. $4)=23.88, p<.01$, and a slight but not statistically significant 


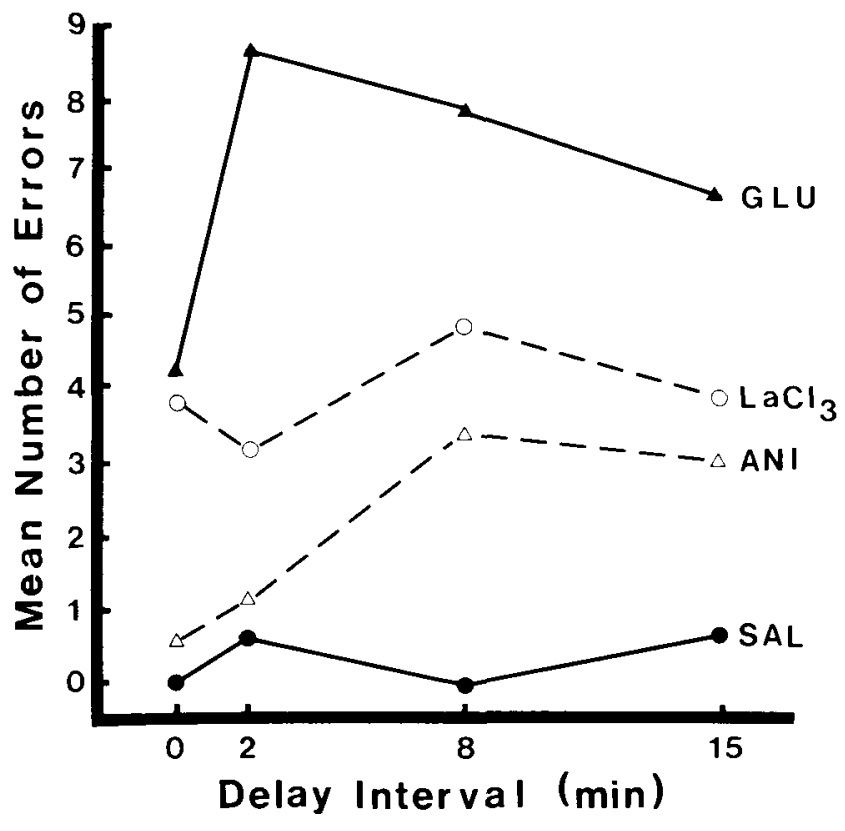

Figure 2. Total number of errors made before rats entered all 12 arms at least once. [Lanthanum $\left(\mathrm{LaCl}_{3}\right)$, glutamate (GLU), anisomysin (ANI), or saline (SAL) were injected $30 \mathrm{~min}$ before Choice 1 . Delays of various lengths were imposed between Choices 6 and 7 . The time course of effectiveness of the different drugs correspond to the data presented in Figure 1.]

interaction of A.VI injection and delay interval, $F(3,12)=$ 3.03, $p<.10$. Examination of Figure 2 shows that more errors were made when 8 - or 15 -min delays followed ANI injection.

It is interesting to note that the extent of performance deficil produced by ANI is similar to the ANI-induced deficit described by Mizumori, Rosenzweig, and Bennett (1985), who trained rats without confinement to the center. Apparently, the confinement procedure used in our experiment per se did not exacerbate the ANI-induced deficit. In both cases, choice accuracy fell to about $70 \%$ after ANI injection, a level of performance that was still considerably above chance.

Comparisons between the effects of GLU or $\mathrm{LaCl}_{3}$ and $\mathrm{SAL}$ also revealed significant effects of drug treatment, $F(1,4)=$ $106.71, p<.001$, and $F(1,4)=20.00, p<.025$, respectively. No significant interaction effect between drug injected and delay interval was found. $F(3,12)=0.65, p>.05$ and $F(3$. 12) $=0.21, p>05$, respectively.

The pattern of results of Experiment 1 suggests two interpretations of the effects of $\mathrm{LaCl}_{3}$ and $\mathrm{GLU}$ on memory over a delay. First, $\mathrm{LaCl}_{3}$ and GLU may have effectively blocked a shorter term component of working memory than did ANI. Alternatively, reference memory may have been impaired by $\mathrm{LaCl}_{3}$ and $\mathrm{GLU}$ in such a way that information regarding associations between environmental cues were not available to interact with information regarding the selection of arms on the day of injection (i.e., working memory items). Because choice accuracy was not altered by ANI when short delay intervals were used, it appears that reference memory was less affected by ANI than was working memory. However, to more clearly ascertain whether $\mathrm{LaCl}_{3}$, GLU. or ANI operated differentially on working and reference memory svstems, one needs to be able to quantify reference memory function more precisely than was possible in Experiment 1 . Therefore, in the following experiment we used changes in the training procedure that were intended to allow simultaneous quantification. within the same rat, of working and reference memory functions after drug injection.

\section{Experiment 2}

\section{Method}

Fourteen rats were trained to retrieve food on a 12-arm radial maze according to the same confinement procedure of Experiment 1 with one exception. In order to improve quantification of working versus reference memory function after drug injection. our training protocol included a subset of arms that were never baited. The set of 8 baited and 4 unbaited arms remained the same for a given rat over the course of the experiment. Different subsets of baited and unbaited arms were used for different rats. The use of this procedure was hased on the suggestion of Olton. Becker, and Handelmann (1979) that accuracy on the baited arms reflects working memory function tin a similar manner as described in Experiment 1) whereas accurate performance on the unbaited arms (1.e.. not entering the unbaited arms) reflects reference memory operations. This procedure has been used in several investigations to test whether experimental manipulations might differentially affect working and reference memories (c.g.. Beatty. Bierley. \& Boyd, 1984). Once rats performed al critcrion levels for 7 days in a row, progressively longer delays (up to $120 \mathrm{~min}$ ) were placed between Choices 4 and 5 . Longer delays of 240 min were not used because, in all of our previous experiments. the drug effects after 120-min delays were similar to those after 240 -min delays.

When the rats achieved criterion performance with 120 -min delays after cannula implantation. the drug-testing phase of the experiment began. $\mathrm{LaCl}_{3}$. GLU. ANI. or SAL was injected $30 \mathrm{~min}$ before Choice 1. All rats were tested after 120 -min delays only. The order of drug administration was determined as described in Experiment 1 . Before another agent (including $S A L$ ) could be injected. the rat was required to perform at criterion levels for 3 days in a row.

\section{Results and Discussion}

Learning not to enter the never-baited arms was surprisingiy difficult for the rats. Figure 3 shows that during the first 10 days of training. rats made more errors to unbaited than to baited arms, although there were only half as many unbaited as baited arms. A repeated-measures ANovs revealed that the numbers of errors to unbaited versus baited arms were significantly different, $F(1,13)=46.84, p<.001$. Both baited and unbaited arm errors declined significantly over the first 10 days of training, $F(9,117)=6.07 . y<.025$. which indicates that significant learning took place for both memory aspects of the task. No significant interaction between days of training and the type of errors made was found, $F(9.117)=0.85, \mathrm{n}>$ .05 . Even when the rats achieved criterion levels of performance with 120 -min delays, more unbaited arm errors $(\boldsymbol{M}=$ 0.39 . $S E= \pm 0.05)$ were made than baited arm crrors $(M=$ $0.06 . S E= \pm 0.02)$.

After surgery, the data were analyzed for 9 rats. Data from 5 rats were eliminated from these analyses because they did 


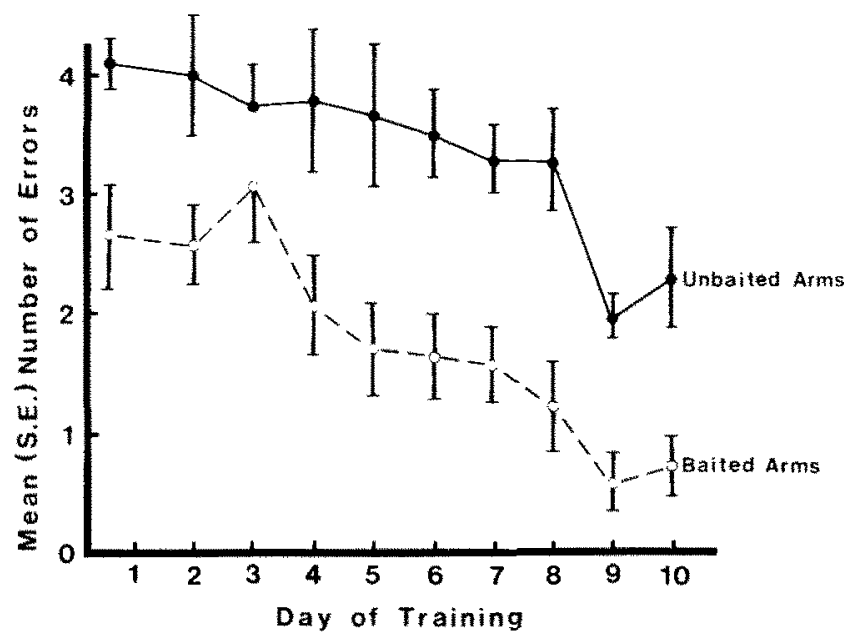

Figure 3. Comparison between the mean number of errors made on baited and unbaited arms during the first 10 days of training. These data indicate that whereas both types of errors significantly declined in number as training progressed, the rats tended to make more errors on unbaited than on baited arms. This was also true for rats performing at asymptotic levels of performance.]

not perform at criterion levels after surgery under nondrugged condition. The presurgery data from these 5 rats did not differ from the data of the remaining 9. The same relationship between types of error made was upheld: Few errors were made overall, and rats still made signilicantly more errors to unbaited than to baited arms.

Histological examinations carried out at the end of the experiment revealed all rats had cannulae placed within ventral hippocampus. The tips of the cannulae were within 0.3 $\mathrm{mm}$ of the CA3 subfield. When we analyzed the effect of $\mathrm{LaCl}$ injection on the number of baited and unbaited arm errors, we found that $\mathrm{LaCl}_{3}$ injection resulted in a slight but not statistically significant increase in baited arm errors made in the first eight choices of the session. $F(1,16)=1.54, p>$ .05 . and a nonsignificant change in the total number of unbaited arm errors, $F(1,16)=2.33, p>.05$ (see upper portion of Figure 4). Upon examining the number of baited and unbaited arm errors made before the delay (see bottom half of Figure 4), we found that most of the errors to unbaited arms occurred during predelay choices; $\mathrm{LaCl}_{3}$ injection resulted in a mean of $0.89(S E= \pm 0.31)$ unbaited arm errors during Choices $1-4$. This was significantly more than the complete absence of such errors during Choices $1-4$ after SAL injection, $F(1.16)=5.44, p<.05$. During the postdelay test period, the number of unbaited arm errors did not differ between $\mathrm{SAL}$ and $\mathrm{LaCl}_{3}$ conditions. Error made on baited arms after $\mathrm{SAL}$ or $\mathrm{LaCl}_{3}$ injections occurred exclusively after the delay interval. $\mathrm{LaCl}_{3}$ produced a slight but not statistically significant increase in the number of baited arm errors after the delay, $F(1.16)=2.07, p>.05$.

GLU injection produced an overall increase in the number of baited but not unbaited arm ertors, $F(1,16)=5.47, p<$ .05 and $F(1,16)=1.93 . p>.05$ (see upper portion of Figure 4). Upon examining errors made before the delay, we found

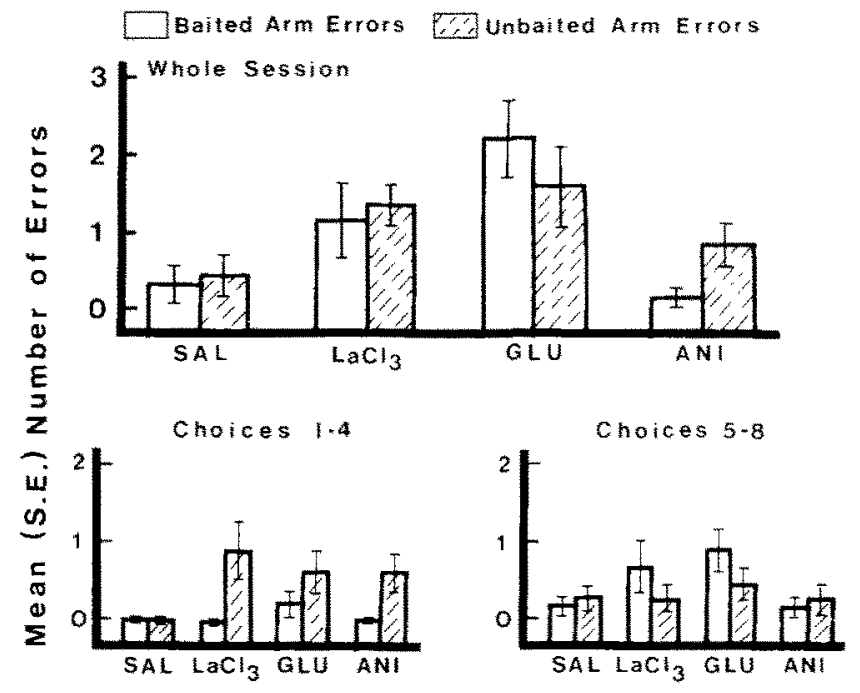

Figure 4. Top half: Maan number of errors made before the rat obtained all food pellets from the maze $(n=9)$. Bottom half: Mean number of errors before (left) or after (right) a 120-min delay. [Two patterns of effects emerged: After lanthanum $\left(\mathrm{LaCl}_{3}\right)$ or glutamate (GLU) injections, there were significantly more unbaited arm errors before the delay; after the delay, the errors tended to occur on baited arms. The second pattern of effect was observed after anisomysin (ANI) injections. Under this condition. more unbaited arm errors occurred before the delay, whereas no imparment on baited or unbaited arms of the maze were observed after the delay SAT = saline.

that in comparison with SAL-injection errors, slight but not statistically significantly more unbaited arm errors were made before the delay when rats were injected with GLU $(M=$ $0.56, S E= \pm 0.24$ ). The number of unbaited arm errors made after the delay did not differ between GLU and SAL conditions. However, there was a slight, though not statistically significant, increase in baited arm errors after the delay on days when rats were injected with GLU. $F(1,16)=3.53 . p>$ .05 .

Like $\mathrm{LaCl}_{3}$ and $\mathrm{GLU}$ injections, $\mathrm{ANI}$ injection also resulted in more unbaited arm errors before the delay $\left(M=0.56 . S F^{\circ}\right.$ $= \pm 0.18), \Gamma(1,16)=6.04, p<.05$, but not after the delay. The number of baited arm errots after $A N I$ injection was identical to that after SAL injection. both before and after the delay. For all drugs tested, performance on the maze $24 \mathrm{hr}$ after injection was nearly perfect. Apparently neither working nor reference memory was permanently impaired. In summary, then, two general patterns of drug effects emerged in this experiment: (a) After $\mathrm{LaCl}_{3}$ or GLU injections. there were significantly more unbaited arm errors before the delay. and after the delay, the errors occurred on baited arms; (b) after ANl injection, more unbaited arm errors were observed before the delay, whereas no impairment on baited or unbaited portions of the task were observed after the delay.

\section{General Discussion}

We investigated the consequences of $\mathrm{LaCl}_{3}$, glutamate. and anisomycin application to hippocampus on working and ref- 
erence memory function in rats. In Experiment $1, \mathrm{LaCl}_{3}$ or GLU injection resulted in poor choice accuracy on the radial maze even when no delay was imposed between choices. ANI injection did not impair retention over relatively short delay intervals (i.e., less than $8 \mathrm{~min}$ ), but impairment was observed if the delay interval was increased to $8 \mathrm{~min}$ or longer. In Experiment 2, in which 4 of the available 12 arms of the radial maze were never baited with food, rats injected with $\mathrm{LaCl}_{3}$ or GLU made significantly more unbaited arm errors before a 120-min delay interval; few unbaited arm entries were observed. but more baited arm errors occurred after the delay. In comparison, injection of ANI produced an increase in the number of unbaited arm errors before the delay with no effect on either type of error after the delay.

The following discussion of these lindings was guided by the premise that whereas performance on unbaited arms requires only an intact reference memory, performance on baited arms probably requires both intact working and reference memories. Furthermore, there were some differences between the memory requirements of the two maze training procedures used in Experiments 1 and 2: The working memory component of the training procedure in Experiment 2 is probably similar to that of Experiment 1, except that fewer arms had to be remembered over the delay period. Reference memory, on the other hand, involved remembering more rules in Experiment 2 than in Experiment 1; that is, rats trained in Experiment 2 had not only to remember the rules necessary for solving the baited arm portion of the maze and associations between its movements in relationship to environmental cues but also to distinguish between baited and unbaited arms, in terms of both their differential significance and the different appropriate responses to be made when each type of arm is encountered. It may even be that different reference memories are necessary to support conect baited and unbaited arm performance.

The effects of $\mathrm{LaCl}_{3}$ and GLU injections were similar to each other for both Experiments 1 and 2. Results of Experiment 1 indicated these drugs impaired performance but did not reveal whether this resulted from interference with a shortterm component of working memory or from interference with reference memory processes. When GLU or $\mathrm{LaCl}_{3}$ was injected into rats of Experiment 2, significantly more unbaited arm errors were made before the delay. This finding supported the hypothesis that $\mathrm{LaCl}_{3}$ and GLU injection interfered with retrieval of reference memory processes, which, perhaps in turn, could have influenced the accuracy with which working memory items were encoded. If the latter was the case, one would expect performance on the baited arm portion of the maze to be impaired before and after the delay period. In fact, however, baited arm errors were made only after the delay.

To explain the finding that relatively few baited arm errors were made before the delay, one might consider the following possibilities: (a) If the rat were selecting arms randomly (during the beginning of the session), there would be a greater probability of making unbaited arm errors than baited arm errors because a first entry into an unbaited arm would be considered an erroneous choice whereas a first entry into a baited arm would be considered a correct choice. As the session proceeds (i.e., after the delay), and if the rat is still selecting arms randomly, the probability of making an error to baited arms increases, whereas the probability of making errors to unbaited arms remains the same. $\mathrm{LaCl}_{3}$ and GLU injections resulted in fewer unbaited arm errors after the delay in comparison with before the delay. This suggests that rats may have been selecting arms randomly before the delay but not after the delay. (b) Because the delay interval was always placed after the fourth choice in the training session (regardless of the number of errors made), the increase in unbaited arm errors before the delay necessarily meant that the number of baited arm choices would be reduced in comparison with control choices. The increased number of unbaited arm errors therefore reduced the probability of making baited arm errors by chance alone during Choices $1-4$. Perhaps if more choices were allowed before the delay, we would have observed an increase in predelay baited arm errors along with the increased number of unbaited arm errors. If the reference memory deficits that occurred before the delay precluded nermal working memory function. one would expect working memory errors after the delay because the rats would not have remembered which arms they entered before the delay. This indeed is what we found.

That more reference memory errors were not found after the delay may reflect the fact that physiological effects of the drugs had worn off. Although there is no direct evidence available to indicate how long the $\mathrm{LaCl}_{3}$ or GI.U injected in these experiments was effective in the rodent brain, there is indirect evidence that the memory-impairing effects of these agents are short-lived. In chicks, Gibbs et al. (1979: Gibbs \& $\mathrm{Ng}, 1979$ ) found that there is a narrow time period around the time of training during which one could administer these agents and observe amnesia. The impairment of reference memory by $\mathrm{LaCl}_{3}$ or GLU was apparently not permanent because (a) one could inject these drugs during the delay period and find no effect on retention when tested after the delay (Mizumori. Channon, et al., 1985) and (b) performance on the maze (with long delay intervals) was nearly perfect 24 hr after injection. However, that $\mathrm{LaCl}_{3}$ and GLU did affect reference memory at all at least indicates a limitation to Gibbs and Ng's hypotheses that these drugs affect only short-lasting memories.

The conclusion that GLU may be affecting reference memory may be more compatible with the results of a study by Morris, Anderson, Lynch, and Baudry (1986). They found that the glutaminergic system may play an important role in reference memories that are involved in place learning. However, the specific findings of Morris et al.'s study are not completely consistent with our results. Factors such as different method of injection, the different approach used to assess reference memory, and the (uncertain) comparability of the drugs used may all have contributed to the different results.

The pattern of errors made after $\mathrm{ANI}$ injection in Experiment 2 was different from that after $\mathrm{LaCl}_{3}$ and GLU as well as unpredicted by the results of Experiment 1 . On the basis of a perhaps less direct method of assessing reference memory function, previous experiments from this laboratory and the results of our Experiment 1 led us to expect that performance on unbaited arms would not be affected by ANI, which would 
thus indicate that reference memory was not affected by ANI. In those particular experiments, ANI-treated rats not only appeared to remember the rules of the task but were able to perform correctly on the maze if a short enough delay interval was used. In Experiment 2, however, we clearly demonstrated that at least some reference memories (as demonstrated on the radial maze) can be affected by ANI. That no impairment on unbaited arms was observed after the delay probably reflects the fact that the physiological effects of ANI were less after the delay than before the delay. In regard to the biochemical effects of ANI, we have evidence to suggest that in rats. protein synthesis inhibition begins to decline significantly by $2 \mathrm{hr}$ after injection (Mizumori, 1985). The finding of unbaited arm errors after A.NI injection is not necessarily contradictory to our previous conclusion because, as noted earlier, there are obvious differences in the reference memory requirements of the tasks used in Experiments 1 and 2. Although the differences in the specific cognitive requirements of the two procedures are not yet well defined, it may be that different reference memories were required to solve both baited and unbaited portions of the maze. If this were true. $\Lambda \mathrm{NI}$ appeared to affect a reference memory responsible for unbaited arm performance and not the one that contributes to performance on baited arms. The necessary usage of different reference memory systems may have influenced the quality and/or quantity of involvement of different neurochemical systems required for solving the maze as a whole. To determine exactly why this should be the case would require additional investigations into how different training procedures might determine the specific neurochemical processes to be involved in initial learning and the subsequent utilization of stored information.

A second point of interest regarding ANI's effects on performance in Experiment 2 is that unlike $\mathrm{LaCl}_{3}$ or $\mathrm{GLU}$ (which also impaired performance on unbaited arms before the delay), it had no effect on choice accuracy for baited arms after the delay. One explanation for this finding could be that protein synthesis is less important in tasks in which the number of working memory items to be remembered is less than that remembered in Experiment 1 ; that is, the effects of ANI on baited arm performance may depend on the relative number of items to be remembered. This most parsimonious explanation is probably not adequate. In a recent experiment. Mizumori, Channon, Rosenzweig. and Bennett (1987) found that ANI was effective in producing an impairment in retention after a 120-min delay in a two-choice delayed alternation task. If one condition for the use of protein synthesis in memory formation were a minimal amount of information to be remembered, then ANI probably would not have had the effect that it did in the relatively simple delayed alternation task. It appears then that the role of protein synthesis in working memory, as in reference memory. may be dependent on the specific cognitive requirements of the overall task. If one aspect of the task. such as the reference memory component of the task used in Experiment 2 in relation to that of Experiment 1 . is made more difficult. the specific constellation of neurochemical events may be altered. This could ultimately lead to a change in the relative contribution of any one particular chemical system.
Our data suggest that the neurochemical systems thought to be involved in memory formation of chicks (Gibbs \& Ng. 1977) do in fact play an important part in spatial memory of rats. However, we found that the characteristics of the amnesic effect produced by drugs used in Gibbs and $\mathrm{Ng}$ 's experiments are specific to the type of memory being tested. This specificity restricts the usefulness of the Gibbs and $\mathrm{Ng}$ sequential memory model (also see Mizumori. Sakai. Rosenzweig. Bennett. \& Wittreich. 1987). Furthermore, we conclude that the exact nature of the involvement of the chemical systems studied here may vary. depending on the specific cognitive (e.g. memory) requirements of the task.

\section{References}

Barroco. D. A.. Lovell. K. L.. \& Eisenstein. E. M. (1981). Effects of cycloheximide and puromycin on learning and retention in the cockroach, P. Americana. Pharmacology, Biochemistry and Beha'ior. 15, 489-494.

Beatty. W. W.. Bierley. R. A.. \& Boyd. J. (1984). Amphetamine disrupts both working and reference memories of rats trained in a radial maze. Behavioral and Neural Biology. 42, 169-176.

Cohen. H. D., \& Barondes. S. H. (1968). Effect of acetoxycycloheximide on learning and memory of a light-dark discrimination. Nature, 218,271-273.

Daniels. D. (1972). Effects of acetoxycycloheximide on appetitive learning and memory. Quarterly Journal of Experimental P. wholog: 24, 102-114.

Davis. H. P.. Idowu, A.. \& Gibson. G. E. (1983). Improvement of 8arm maze performance in aged Fischer 344 rats with 3.4-diaminopyridine. Experimental Aging Rexearch, 9.211-214.

Davis, H. P., \& Squire, L. R. (1984). Protein synthesis and memor: A review. Pswchological Bulletin. 96, 518-559.

Gibbs, M. E.. Gibbs, C. L.. \& Ng. K. T. (1978). Possible physiological mechanism for short-term memory. Phisiology and Behavior. 20. 619-627.

Gibbs, M. E.. Gibbs. C. L.. \& Ng. K. T. (1979). The influence of calcium on short-term memory. Neurosctence Letters. 14. 355360 .

Gibbs. M. E. \& Ng. K. T. (1976). Memory formation: A new threcphase model. Neuroscience Letters, 2, 165-169.

Gibbs, M. E., \& Ng. K. T. (1977). Psychobiology of memory: Towards a model of memory formation. Biobehavioral Reviews. /. 113136.

Gibbs, M. E., \& Ng. K. T. (1979). Neuronal depolarization and the inhibition of short-term memory formation. Physioligy and $B e^{-}$ havior. 23, 369-375.

Gibbs. M. E.. \& Ng, K. T. (1984a). Diphenylhydantoin extension of short-term and intermediate stages of memory. Beharioural Brain Research, 11, 103-108.

Gibbs. M. E.. \& Ng. K. G. (1984b). Dual action of cycloheximide on memory formation in day-old chicks. Behavioural Brain Resear h. 12. $21-27$.

Handelmann, G. E., \& Olton, D. S. (1981). Spatial damage to hippocampal CA3 pyramidal cells with kainic acid: Impairment and recovery with preoperative training. Brain Researd $h, 217.41-$ 58.

Mizumori, S. J. (1985). Neurochemical models of memem: Unpublished doctoral dissertation. University of Califomia at Berkeles.

Mizumori, S. J. Y.. Channon, V.. Rosenzweig. M. R. \& Bennett. E. L. (1985). The involvement of calcium, glutamate, and protein synthesis in short- and long-term working memories of the rat. Society for Neuroscience Abstracts. 11.872. 
Mizumori, S. J. Y., Channon, V., Rosenzweig, M. R., \& Bennett, E. L. (1987). Anisomycin impairs long-lerm working memory in a delayed alternation task. Behavioral and Neural Biology, 47, 1-6.

Mizumori, S. J. Y., Rosenzweig, M. R., \& Bennett, E. L. (1985). Long-term working memory in the rat: Effects of hippocampally applied anisomycin. Behavioral Neuroscience, 99, 220-232.

Mizumori, S. J. Y., Sakai, D. H., Rosenzweig. M. R., Bennett, E. L., \& Wittreich, P. (1987). Investigations into the neuropharmacological basis of temporal stages of memory formation in mice trained in an active avoidance task. Behavioural Brain Research, 23, 239250.

Montarolo, P. G., Castellucci, V. F., Goelet, P., Kandel, E. R., \& Schacher, S. (1985). Long-term facilitation of the monosynaptic connection between sensory neurons and motor neurons of the gill-withdrawal reflex in Aplysia in dissociated cell culture. Society for Neuroscience Ahstracts, 11, 795 .

Morris, R. G. M., Anderson, E., Lynch, G. S., \& Baudry, M. (1986). Selective impairment of learning and blockade of long-term potentiation by an $\mathrm{N}$-methyl-D-aspartate receptor antagonist, AP5. $\mathrm{Na}$ ture, 319, 774-776.
Olton, D. S. (1978). Characteristics of spatial memory. In S. H. Hulse, H. Fowler, \& W. K. Honig (Eds.), Cognitive processes in animal behavior (pp. 341-373). Hillsdale, NJ: Erlbaum.

Olton, D. S. (1983). Memory function and the hippocampus. In W. Seifert (Ed.), Neurobiology of the hippocampus (pp. 335-373). New York: Academic Press.

Olton, D. S., Becker, J. T., \& Handelmann, G. E. (1979). Hippocampus, space, and memory. Behavioural Brain Sciences, 2, 313-365. Staubli, U., Baudry, M., \& Lynch, G. (1984). Leupeptin, a thiol proteinase inhibitor, causes a selective impairment of spatial maze performance in rats. Behavioral and Neural Biology, 40, 58-69.

Watts, M. E., \& Mark, R. F. (1971). Drug inhibition of memory formation in chickens. II. Short-term memory. Proceedings of the Royal Society: Series B, 178, 455-464.

Received January 20, 1986

Revision received November 5, 1986 Accepted November 7, 1986

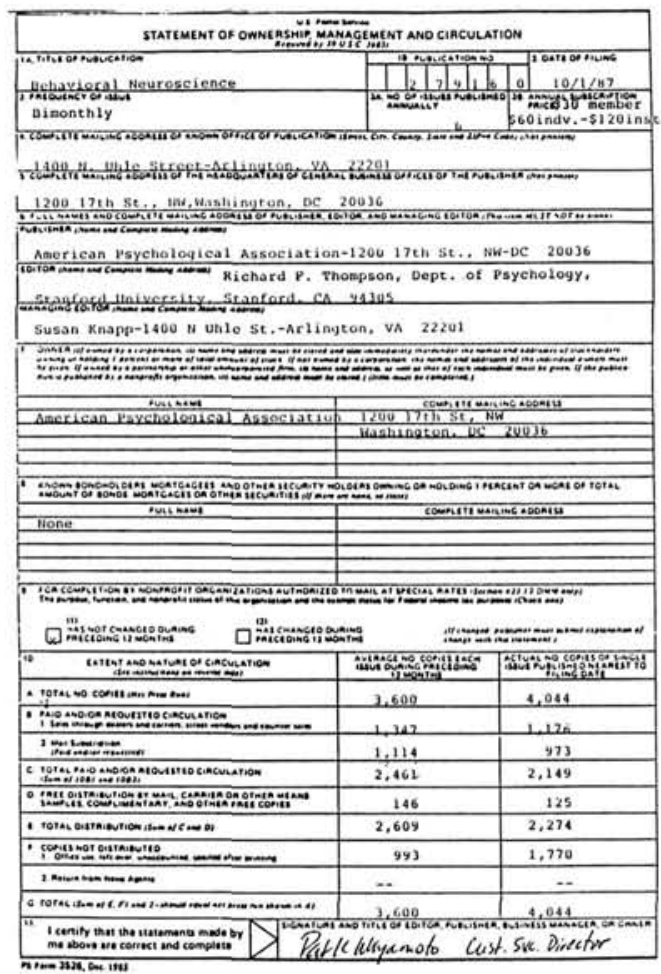

